\title{
Funciones Ejecutivas y Ajuste Clínico en Adolescentes Colombianos Policonsumidores
}

\author{
Executive Functions and Clinical Adjustment in Colombian \\ Adolescents Polydrug
}

\author{
Sandra Milena Camelo Roa \\ Facultad de Psicología. Universidad Católica de Colombia \\ Teresa Olivares Pérez \\ Departamento de Psicología Clínica, Psicobiología y Metodología \\ Universidad de la Laguna, Tenerife-España \\ Mónica Carballeira Abella \\ Departamento de Psicología Clínica, Psicobiología y Metodología \\ Universidad de la Laguna, Tenerife- España \\ Moisés Betancort \\ Departamento de Psicología Clínica, Psicobiología y Metodología \\ Universidad de la Laguna, Tenerife-España
}

Rec (28 de febrero de 2018) Acept (28 de mayo de 2019)

\begin{abstract}
Resumen
El estudio tuvo como objetivo analizar el funcionamiento ejecutivo y el ajuste clínico en una muestra de adolescentes colombianos policonsumidores. Se efectuó un estudio ex post facto con 204 participantes entre 12 y 17 años $(M=14,50, D T=1,71)$. Se aplicó la Batería Neuropsicológica de Funciones Ejecutivas y Lóbulos Frontales BANFE-2 y para la evaluación clínica se utilizó la Escala de Autoestima de Rosenberg, Cuestionario de Afrontamiento COPE, Escala de Impulsividad de Dickman y Cuestionario de 90 Síntomas SCL-90-R. Se identificó un rendimiento significativamente inferior del grupo policonsumidor en las funciones ejecutivas, bajos niveles de autoestima, altos niveles de impulsividad disfuncional y de diversidad psicopatológica, así como estilo de afrontamiento a través del consumo de sustancias. Así mismo, se detectó un efecto predictor de la interacción edad $\mathrm{x}$ tiempo de consumo en el funcionamiento ejecutivo, observándose un menor rendimiento de los policonsumidores más jóvenes a medida que se incrementaba el tiempo de consumo.

Palabras Clave: Policonsumo, Funcionamiento Ejecutivo, Ajuste Clínico, Adolescencia, Tiempo de Consumo.
\end{abstract}

\begin{abstract}
The research aim was to analyze the executive functioning and the clinical adjustment in a sample of Colombian teen polydrug users. An ex post facto study was carried out with 204 participants, $12-17$ years old $(M=14,50, S D=1,71)$. The neuropsychological test BANFE-2 was applied for analyzing frontal lobes and executive functioning and the Rosenberg self esteem scale, a COPE questionnaire, the Dickman impulsivity scale and the SCL-90-R questionnaire were used for clinical adjustment respectively. A lower executive functioning throughput were observed in polydrug users. Low self-esteem, high dysfunctional impulsivity, high diverse psychopathologies and coping strategies for drug use were also observed. A predictor effect between age and consumption time on the executive functioning were also determined. A lower throughput in younger polydrug users increase through consumption time.

Keywords: Polydrug users, executive functioning, clinical adjustment, adolescence and consumption time.
\end{abstract}




\section{Introducción}

El consumo de sustancias psicoactivas, visto como un fenómeno colectivo constituye un gran problema de salud pública que merece ser atendido por el personal de la salud, con el propósito de reducir la incidencia del consumo y los problemas de morbilidad y mortalidad que conlleva. Son múltiples los factores que se han atribuido como causa a la dependencia de las drogas, sin embargo estos no han sido totalmente esclarecidos y deben analizarse desde diferentes perspectivas.

La mayoría de estadísticas mundiales muestran que la adolescencia es la etapa de mayor vulnerabilidad para el inicio del consumo de las drogas. Las cifras nacionales en Colombia muestran un incremento en los últimos años de consumo y una amplia variedad de sustancias usadas en la que no sólo se destacan drogas ilícitas sino también el tabaco y alcohol. En el último Estudio Nacional de Consumo de Sustancias Psicoactivas 2013 (Min Justicia, Min Salud \& ODC, 2013), se revela un claro aumento del consumo global de sustancias ilícitas (marihuana, cocaína, bazuco, éxtasis y heroína) en comparación con 2008. El reporte también refleja aumento de uso en el último año de $2.6 \%$ a 3.6\%. La marihuana continuó siendo la sustancia ilícita de mayor consumo y se confirmó la existencia de un consumo bajo, pero probablemente creciente, de sustancias ilícitas sintéticas como heroína, anfetaminas (éxtasis), inhalables (pegantes, pinturas, popper, dick) y otros (metadona, 2CB, ketamina, GHB) en los adolescentes.

La experimentación con diferentes sustancias durante la adolescencia es común que comience entre los 13 y 16 años de edad, con una o varias sustancias ocasionalmente, sin generar problemas psicosociales significativos. Sin embargo, la gran mayoría de adolescentes que inician el consumo tempranamente continúa consumiendo, hasta constituirse un patrón regular que lo hace característico del trastorno por abuso o dependencia de una o más sustancias, con todas las repercusiones desfavorables que conlleven (Martinez, Amaya, Campillo, Rueda, Campo \& Díaz, 2007).

Los estudios epidemiológicos develan que las características sociodemográficas (nivel socioeconómico, entorno, edad) las personales (problemas emocionales) y la relación con los padres (herencia, disfunción familiar, divorcio) son los factores de riesgo con mayor importancia para el consumo de sustancias psicoactivas (Páramo, 2011; Moral \& Ovejero, 2011; Muñoz, Gallego, Wartski \& Álvarez, 2012; Martinez et al., 2007; Camacho, 2005).

El policonsumo se define como la dependencia de varias sustancias que experimenta un individuo (American Psychiatric Association, 2000). Más concretamente, se aplicaría a aquellos casos en los que, durante un periodo de 12 meses, el sujeto consume repetidamente, como mínimo, tres grupos de sustancias sin que predomine una sobre otra y sin importar al sujeto consumidor la evidencia de consecuencias y afecciones físicas (Mariño, Castro \& Torrado, 2012).

De acuerdo con Flores \& Fernández (2011), la investigación sobre los mecanismos psicobiológicos que sustentan la adicción ha contribuido a un aumento del conocimiento de los sistemas neurales que regulan el proceso de refuerzo, ya sea ante estímulos naturales como artificiales. Para Corominas, Roncero y Casas (2015) el sistema dopaminérgico constituye la principal vía neurobiológica implicada en el fenómeno de la adicción. Según estos autores, las drogas de abuso convergen en un mecanismo neurobiológico común con intervención de los circuitos límbicos del cerebro. Según Rosenberg y Curtiss (2014), las alteraciones en las estructuras mesolímbicas y la corteza frontal medial perpetúan el ciclo adictivo (Corominas et al., 2015; Acosta, Cervantes, Pineda, Torre, López \& Cárdenas, 2011; Blum, Femino, Teitelbaum, Giordano, Berman \& Gold, 2013).

La investigación en este campo también identifica que las limitaciones en el desarrollo de los procesos madurativos cerebrales en la adolescencia, derivadas de desconexiones entre las estructuras cerebrales subcorticales y frontales, incrementan la búsqueda de sensaciones y la incapacidad para controlar impulsos (Konrad, Firk \& Uhlhaas, 2013); así como las conductas de asunción de riesgos, como el consumo de sustancias (Lydon, Wilson, Child \& Geier, 2014). La corteza prefrontal, en pleno desarrollo madurativo al comienzo de la adolescencia, es una de las estructuras cerebrales más frecuentemente vinculada con este tipo de conductas (Oliva, 2007).

El consumo de drogas también se ha relacionado con la existencia de alteraciones en distintos procesos y funciones neuropsicológicas. Frecuentemente, esta relación se ha descrito como el deterioro cognitivo producido por dicho consumo. Sin embargo, en la actualidad existe evidencia empírica que señala que el funcionamiento cognitivo es un factor importante en la adquisición y mantenimiento de la conducta adictiva (Verdejo-García, Bechara, Recknor, \& Perez-Garcia, 2006). En este sentido, las funciones ejecutivas (FE) son relevantes para 
la adaptación social, vigilancia y regulación de la conducta y, en consecuencia, la alteración en estas funciones podría favorecer la manifestación de conductas desadaptativas como la adicción (Inozemtseva, 2013). Investigaciones recientes indican que existe una importante vinculación de las FE con la conducta adictiva (Heitzeg, Cope, Martz \& Haredee, 2015; Wilson, et al., 2015). De acuerdo con Llanero-Luque, Ruiz-Sánchez de León \& Pedrero-Pérez (2014), en las dos últimas décadas se ha acumulado un gran número de estudios que pretende dar cuenta de los cambios que se producen en el cerebro adicto. Fernández-Serrano, Pérez-García \& Verdejo-García (2011) llevaron a cabo una exhaustiva revisión de la mayoría de estudios disponibles en la que se identifica un conjunto común de alteraciones neuropsicológicas asociadas a la adicción, independientemente de la sustancia implicada. Hallaron dificultades en la memoria episódica, alteraciones en el funcionamiento ejecutivo y déficits en el procesamiento emocional.

La investigación sobre personalidad considera el funcionamiento cerebral como sustento de los patrones estables de conducta. Particularmente, la corteza frontal muestra una gran vinculación con estos rasgos estables y sus alteraciones también tienen un reflejo en el desarrollo de ciertos patrones de comportamiento. Algunos autores consideran que los rasgos temperamentales podrían tener mayor implicación que los propios factores ambientales en los patrones de consumo (Beckman et al., 2011). La inestabilidad emocional, la hostilidad, la somatización y el psicoticismo, se identifican como algunos de los rasgos de personalidad asociados al consumo de drogas (Thoma, Friedmann \& Suchan, 2013). Además, la autoestima, el afrontamiento o la impulsividad, pueden influir en la forma en que los adolescentes interpretan las señales del entorno, condicionando la toma de decisiones respecto al consumo (Gullo \& Potenza, 2014).

La literatura presenta investigaciones que han tratado de estudiar la autoestima sobre adolescentes sanos y sobre consumidores de sustancias. Estudios realizados en adolescentes policonsumidores han identificado que el clima familiar, el entorno personal y social, tienen un impacto sobre el desarrollo de la autoestima y el inicio del consumo de sustancias psicoactivas en adolescentes con edades comprendidas entre 12 y 17 años (Sajjadi, Harouni \& Sani, 2015; Sánchez-Sosa, Villarreal-González, Ávila-Guerrero, Vera-Jiménez \& Musitu, 2014).

La impulsividad también puede ser determinante en la búsqueda y experimentación de distintas sensaciones con la finalidad de estimular el sistema de recompensa, lo cual, es representativo en la etapa de la adolescencia y conduce posiblemente al inicio de la conducta de consumo de sustancias (Gullo, Loxton \& Dawe, 2014).

Los estilos de afrontamiento que a menudo utilizan los adolescentes varían en función de unas variables específicas que van a determinar cuán exitoso es un tipo de afrontamiento como solución a un problema determinado. De acuerdo con la literatura, se ha identificado que el consumo de sustancias puede ser un tipo de afrontamiento para evitar emociones propias de la ansiedad y depresión, tal es el caso del consumo de éxtasis, funcionando como un reforzador negativo que ayuda a evitar síntomas depresivos y de ansiedad (Scott, et al., 2013). Los estudios en los que se ha aplicado el Inventario de Estrategias de Afrontamiento - COPE - para identificar modos de afrontar en consumidores de alcohol y otras sustancias, incluyen el "consumo de alcohol y/o drogas", identificando en esta población un mayor uso de dicho estilo de afrontamiento ante eventos estresantes, lo que va unido a un peor pronóstico en la intervención (Bussey, et al., 2006).

En ciertos casos, la adolescencia se caracteriza por la presencia de algunas variables psicopatológicas, hallándose relación entre ideación suicida, depresión y baja autoestima en adolescentes (Rosselló \& Berríos Hernández, 2004). Además, el consumo de sustancias psicoactivas se ha asociado con la presencia de trastornos como ansiedad, depresión, obsesión- compulsión, somatización, hostilidad, ideación paranoide, psicoticismo, entre otros (Lev-Ran, Le Foll, McKenzie, George \& Rehm, 2013; Borges, Bagge \& Orozco, 2016).

La revisión de la literatura asociada al fenómeno del consumo y abuso de drogas, pone de manifiesto un cambio en la conceptualización de la dependencia. En el campo de la neuropsicología de las adicciones, el interés científico se dirigiría a demostrar la implicación de los procesos vinculados a las funciones ejecutivas en la conducta de búsqueda y consumo de drogas. Además, desde el ámbito temperamental, se considera que ciertas variables de personalidad, pueden influir sobre la tendencia al consumo y dependencia de sustancias en los adolescentes. La detección de altas tasas de consumo temprano, justifica la necesidad del estudio de dichos aspectos de manera integrada.

\section{Método}




\section{Participantes}

La muestra la conformó un total de 204 adolescentes varones entre 12 y 17 años de edad seleccionados mediante un muestreo no probabilístico-intencional. El grupo objeto de estudio incluyó 102 participantes provenientes de un centro de orientación juvenil de formación y reeducación para policonsumidores de sustancias psicoactivas. El grupo control se formó con 102 jóvenes no consumidores, provenientes de una institución educativa para estudiantes con escasos recursos socioeconómicos, beneficiarios de subsidios en los servicios públicos domiciliarios en Colombia. Ambos grupos mostraron características demográficas equivalentes en edad, grupo policonsumidor y control $(M=14,50 ; D T=1,71)$, y nivel educativo, grupo policonsumidor $(M=6,45 ; D T=1,63)$, grupo control $(M=8,3 ; D T=1,64)$. Los criterios de inclusión contemplados fueron los siguientes: (a) edad entre 12 y 17 años; (b) ausencia de enfermedades orgánicas que pudieran provocar déficit cognitivo y/o psicopatológico graves; (c) ausencia de incapacidad física para realizar las tareas requeridas; (d) ausencia de deficiencias auditivas o visuales que no estuvieran corregidas y, por lo tanto, que pudieran afectar a la realización de las diferentes pruebas; (e) historia de policonsumo de doce meses mínimo para el grupo policonsumidor; (f) periodo de abstinencia a las sustancias mínimo de uno a tres meses para el grupo policonsumidor.

\section{Instrumentos}

Batería Neuropsicológica de Funciones Ejecutivas y Lóbulos Frontales (BANFE-2) (Flores, Ostrosky \& Lozano, 2014). Es una batería neuropsicológica diseñada para la evaluación de los procesos cognitivos que dependen principalmente de la corteza prefrontal. Evalúa 14 procesos relacionados con las Funciones Ejecutivas (flexibilidad mental, productividad, control inhibitorio, planificación, memoria de trabajo, abstracción, fluidez (verbal), generación de hipótesis, estrategias de trabajo, seriación, secuenciación, metacognición, monitoreo y control,), agrupados en tres áreas de exploración frontal: Órbitomedial, Prefrontal Anterior, y Dorsolateral.

Las pruebas que conforman la batería son ampliamente utilizadas por la comunidad internacional y seleccionadas bajo un procedimiento de validez convergente y clínica propuesto para la neuropsicología (Stuss \& Levine, 2002). Esta batería permite obtener índices parciales para cada una de las áreas evaluadas y un índice global de desempeño.

Alcohol, Smoking and Substance Involvement Screening Test (Versión 3.1.) WHO ASSIST Working Group (2002). Es un cuestionario en versión de papel y lápiz dirigido a los consumidores y que consta de ocho preguntas para detectar el consumo de sustancias como: tabaco, alcohol, cannabis, estimulantes de tipo anfetamina, inhalantes, sedantes o pastillas para dormir (benzodiacepinas), alucinógenos, opiáceos y otras drogas. Proporciona información sobre la frecuencia de consumo de sustancias a lo largo de la vida, así como los problemas relacionados en los últimos tres meses. El cuestionario indica la puntuación de riesgo para cada sustancia que el usuario informa haber consumido (bajo, moderado o alto). El diseño de la prueba es culturalmente neutral, por lo que puede utilizarse en una gran variedad de culturas.

Society and the Adolescent Self-image (Rosenberg, 1965). Es una de las escalas más utilizadas para la medición global de la autoestima en adolescentes. Incluye diez ítems cuyos contenidos se centran en los sentimientos de respeto y autoaceptación. La mitad de los ítems está enunciado positivamente y la otra mitad negativamente. La graduación de respuestas tiene 4 puntos (1=muy en desacuerdo, $2=$ en desacuerdo, $3=$ de acuerdo y $4=$ muy de acuerdo) y se asigna el puntaje inverso a las afirmaciones direccionadas negativamente. Los valores fluctúan entre 0 y 25 (baja autoestima), de 26 a 29 (autoestima normal) y de 30 a 40 (alta autoestima). Las características psicométricas de esta escala han sido ampliamente estudiadas en su versión original, así como en otros idiomas (Vázquez-Morejón, Jiménez-García \& Vázquez-Morejón, 2004), mostrando buenos indicadores psicométricos.

Coping Estimation Questionnaire (COPE) (Carver, Scheier \& Weintraub, 1989). Es un inventario multidimensional de 60 ítems compuesto por 15 subescalas que hacen referencia a los distintos modos de afrontar el estrés, evaluando la frecuencia con que las personas utilizan las siguientes estrategias: Búsqueda de apoyo social, Religión, Humor, Consumo de alcohol y/o drogas, Planificación y afrontamiento activo, Abandono de los esfuerzos de afrontamiento, Centrar esfuerzos para solucionar la situación, Crecimiento personal, Reinterpretación positiva, Actividades distractoras de la situación y Evasión. La puntuación para cada una de ellas oscila entre 1 y 4. Las propiedades psicométricas del cuestionario han sido ampliamente probadas (Todd, Tennen, Carney, Armeli \& Affleck, 2004). Para este estudio se ha utilizado la versión española realizada por Crespo \& Cruzado 
(1997).

Dickman Impulsivity Inventory (DII) (Dickman, 1990). Consta de 23 ítems divididos en dos escalas: once miden Impulsividad Funcional y doce Impulsividad Disfuncional. Las respuestas son de elección forzosa en sentido afirmativo o negativo. Recientemente se ha validado en población drogodependiente una versión modificada, más fiel a la original (Pedrero-Pérez, 2009) que mostró adecuados valores de consistencia interna (alfa 0,86 y 0,74 para ambas escalas, respectivamente).

\section{Procedimiento}

Una vez seleccionada la muestra en ambas instituciones, se organizó en 6 grupos formados por 17 adolescentes cada uno de ellos, correspondientes a los grupos de edades contempladas en el estudio. La evaluación se llevó a cabo de manera individual durante 10 meses, utilizando el mismo protocolo de evaluación para ambas muestras (grupo de policonsumidores y grupo control), en dos sesiones de hora y media cada una, aplicadas en días diferentes y con el siguiente orden: En la primera sesión se suministró explicación a cada uno de los participantes las condiciones del estudio, el tratamiento ético de los datos y firma de consentimiento informado; seguidamente se administró la historia clínica para la obtención de los datos sociodemográficos y la aplicación de la prueba de detección de consumo de sustancias (ASSIST). Posteriormente en la segunda sesión, se llevó a cabo la evaluación clínica, con el siguiente orden: a) Escala de Deseabilidad Social del EPQ-RS; b) Inventario de Impulsividad (DII); c) Cuestionario de Estrategias de Afrontamiento (COPE); d) Escala de Autoestima y e) Inventario de Sintomatología Psicopatológica (SCL-90-R). En esta sesión se realizó la evaluación neuropsicológica de las funciones ejecutivas mediante la Batería Neuropsicológica de Funciones Ejecutivas y Lóbulos Frontales (BANFE-2), respetando el orden establecido del protocolo de evaluación de la batería: a) Laberintos; b) Señalamiento autodirigido; c) Ordenamiento alfabético de palabras; d) Resta consecutiva; e) Suma consecutiva; f) Clasificación de Cartas; g) Clasificaciones semánticas; h) Efecto Stroop, forma A; i) Fluidez Verbal; j) Juego de cartas; k) Selección de refranes; 1) Torre de Hanoi; m) Metamemoria; n) Memoria de trabajo visoespacial; y ñ) Efecto Stroop, forma B.

El estudio se realizó bajo la normativa ética requerida en la investigación con seres humanos bajo los principios de la Declaración de Helsinky.

\section{Análisis de datos}

Para el presente estudio se llevaron a cabo análisis estadísticos multivariados con el lenguaje de programación de análisis de datos R (R Development Core Team, 2008). Previamente a los análisis, se llevó a cabo un estudio de casos extremos multivariados. El criterio utilizado fue el estadístico $\mathrm{D}^{2}$ de Mahalanobis como índice de distancia multivariada al centroide de la distribución para cada registro de la base de datos, considerando tanto el grupo policonsumidor como el control. Los datos excluidos lo fueron con el criterio de una significación al $1 \%$. Posteriormente se realizó el análisis discriminante con el objetivo de observar la combinación lineal de variables que separaban ambos grupos de una manera más eficaz. Esto se llevó a cabo tanto para las variables neuropsicológicas como clínicas. El resultado permitió generar un modelo multivariado que mostró su efecto en la función ejecutiva y el ajuste clínico, tanto en el grupo control como policonsumidor. Una vez analizadas las diferencias en las variables neuropsicológicas y clínicas entre los grupos, se estudió cómo se relacionan estas variables a la hora de clasificar a los sujetos en sus respectivos grupos; para ello, se llevó a cabo un análisis Random Forest (RF) (Breiman, 2001). Este análisis es una técnica de clasificación guiada por los datos que permite seleccionar aquéllas variables cuyas puntuaciones generan grupos con valores muy cercanos entre ellos y distantes con respecto al otro. Una vez realizado este análisis, se establecieron los puntos de sensibilidad a partir de las curvas ROC para las variables seleccionadas e identificar al grupo policonsumidor. Finalmente se realizó un análisis de regresión múltiple paso a paso teniendo en cuenta las puntuación total de la batería de funciones ejecutivas BANFE-2, siendo las variables predictoras la edad y el tiempo de consumo, así como el término de interacción Edad*Tiempo de Consumo.

\section{Resultados}


Los resultados del análisis discriminantes para variables neuropsicológicas mostró una función significativa de separación máxima de los grupos $[\lambda=0,51, \mathrm{~F}(1,201)=190,144, \mathrm{p}<0,05]$. La relación de la función aislada con los grupos fue de 0,69 (correlación canónica). Las variables con más peso a la hora de separar al grupo control del grupo policonsumidor fueron BANFE-2 total prefrontal, BANFE total dorsolateral y BANFE-2 total funciones ejecutivas (FE); estas variables mostraron coeficientes de estructura mayores (Tabla 1).

Tabla 1. Coeficientes de estructura, típicos y medias para las variables discriminantes neuropsicológicas

\begin{tabular}{lcccc}
\hline \multicolumn{1}{c}{ Matriz de coeficientes } & \multicolumn{2}{c}{ Función 1 } & \multicolumn{2}{c}{ Medias } \\
& Estructuras & Típicos & Control & Policonsumidor \\
\hline Banfe Total Prefrontal & 81,70 & 64,12 & 20.129 & 15.873 \\
Banfe Total Dorsolateral & 68,51 & 41,73 & 20.653 & 163,48 \\
Banfe Total Funciones Ejecutivas & 71,80 & 26,45 & 415.891 & 359.196 \\
\hline
\end{tabular}

Al observar el espacio discriminante, se interpreta el efecto multivariado que esta función aislada y las variables que la componen tienen sobre la separación de los grupos (ver figura 1). La inspección de este espacio discriminante nos permitió observar cómo la función 1 (LD1) separó con una máxima eficacia al grupo control del grupo policonsumidor. Se observó que el grupo policonsumidor obtiene puntuaciones positivas en la función, que reflejarían un menor rendimiento en las distintas subpruebas del BANFE-2 con respecto al grupo control (Tabla 1).

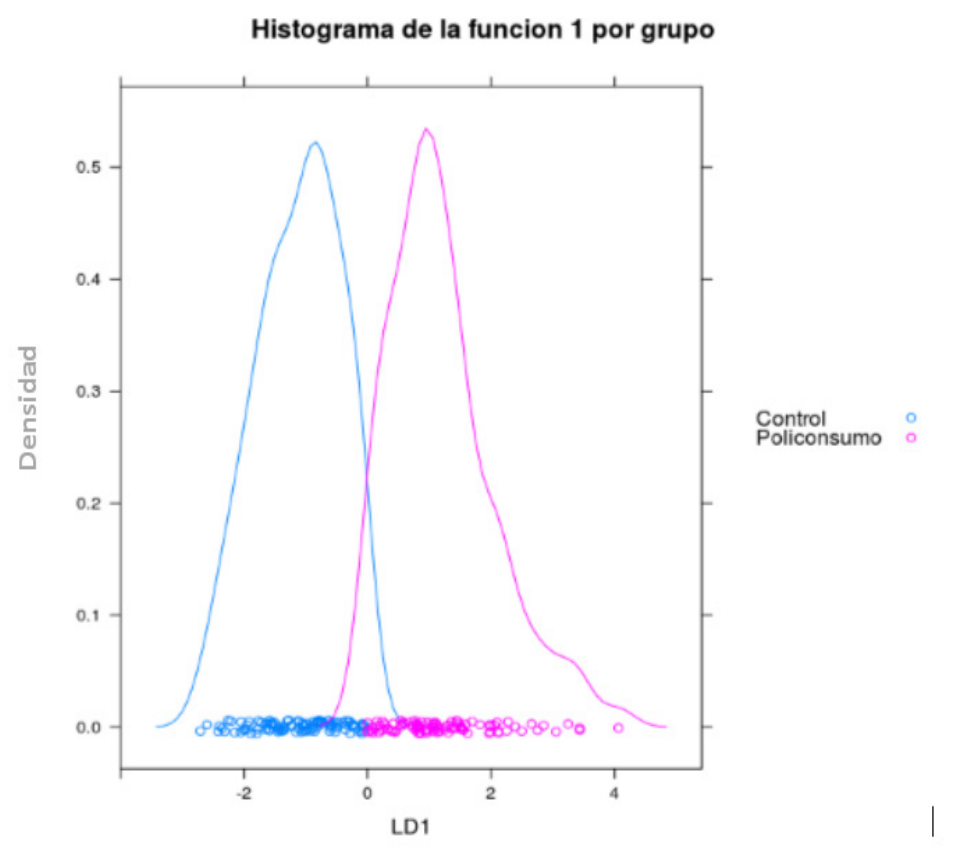

Figura 1. Espacio discriminante para variables neuropsicológicas 
En relación al análisis discriminante para variables clínicas el resultado mostró una función significativa de separación máxima de los grupos $[\lambda=0,47, \mathrm{~F}(1,201)=220,9559, \mathrm{p}<0,05]$. La relación de la función aislada con los grupos fue de 0,72 (correlación canónica). Las variables con más peso a la hora de separar al grupo control del grupo policonsumidor fueron: Afrontamiento con consumo de alcohol y drogas (COPE 4), Impulsividad funcional, Impulsividad disfuncional, Autoestima e Índice de amplitud y diversidad psicopatológica (SCL90) (Tabla 2).

Tabla 2. Coeficientes de estructura, típicos y medias para las variables discriminantes Clínicas

\begin{tabular}{lcccc}
\hline \multirow{2}{*}{ Matriz de coeficientes } & \multicolumn{2}{c}{ Función 1 } & \multicolumn{2}{c}{ Medias } \\
& Estructuras & Típicos & Control & Policonsumidor \\
\hline Afrontamiento Consumo de Alcohol o drogas & 81,70 & 64,12 & 20.129 & 15.873 \\
Impulsividad disfuncional & 68,51 & 41,73 & 20.653 & 163,48 \\
Amplitud y diversidad psicopatológica & 71,80 & 26,45 & 415.891 & 359.196 \\
\hline
\end{tabular}

La inspección del espacio discriminante para variables clínicas permitió observar cómo la función 1 (LD1) separa con una máxima eficacia al grupo control del grupo policonsumidor (Figura 2). Se pudo observar que este último muestra puntuaciones positivas en la función, en contraste con el grupo control (Tabla 2), lo que implicaría un peor rendimiento en las variables Afrontamiento consumo de alcohol o drogas (COPE 4), Impulsividad disfuncional, Índice de amplitud y diversidad psicopatológica (SCL90-PST).

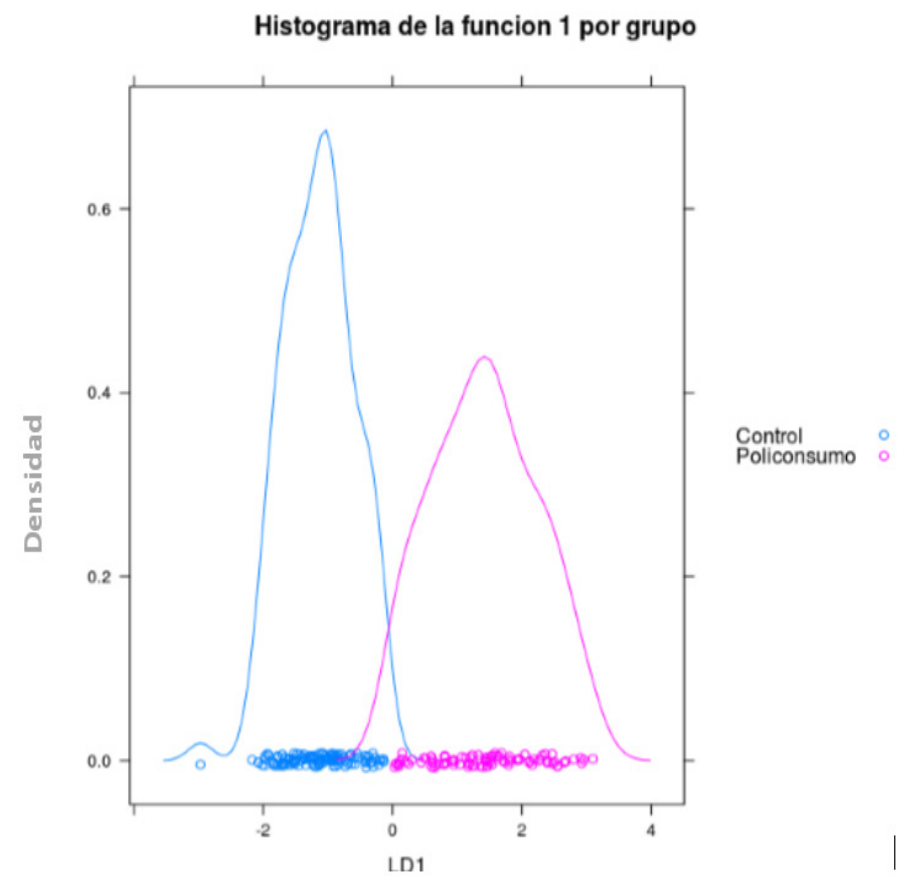

Figura 2. Espacio discriminante para variables clínicas 
El análisis Ramdom Forest mostró una solución a partir de 5000 árboles usando 3 variables en cada división con un error estimado (error OOB) de tan sólo del 9,85\%, representado en una matriz de confusión en el que el mejor grupo clasificado fue el grupo control, frente al grupo de policonsumidores. Asumiendo este porcentaje de error de clasificación en el análisis iterativo al azar resultaron seleccionadas tres variables: Estrategia de afrontamiento consumo de alcohol o drogas (COPE 4), BANFE total dorsolateral, BANFE total prefrontal. Tal y como se observa en la figura 3, una puntuación en el COPE 4 menor a 1 y una puntuación en la BANFE dorsolateral > a 186 reflejaría la combinación de variables que con mayor probabilidad pronostica la pertenencia al grupo control. Por otro lado, una puntuación del COPE 4 mayor a 1 y una puntuación en el BANFE prefrontal menor o igual a 21 junto a una puntuación del BANFE dorsolateral menor a 203, sería la combinación que mejor pronosticaría la pertenencia al grupo policonsumidor.

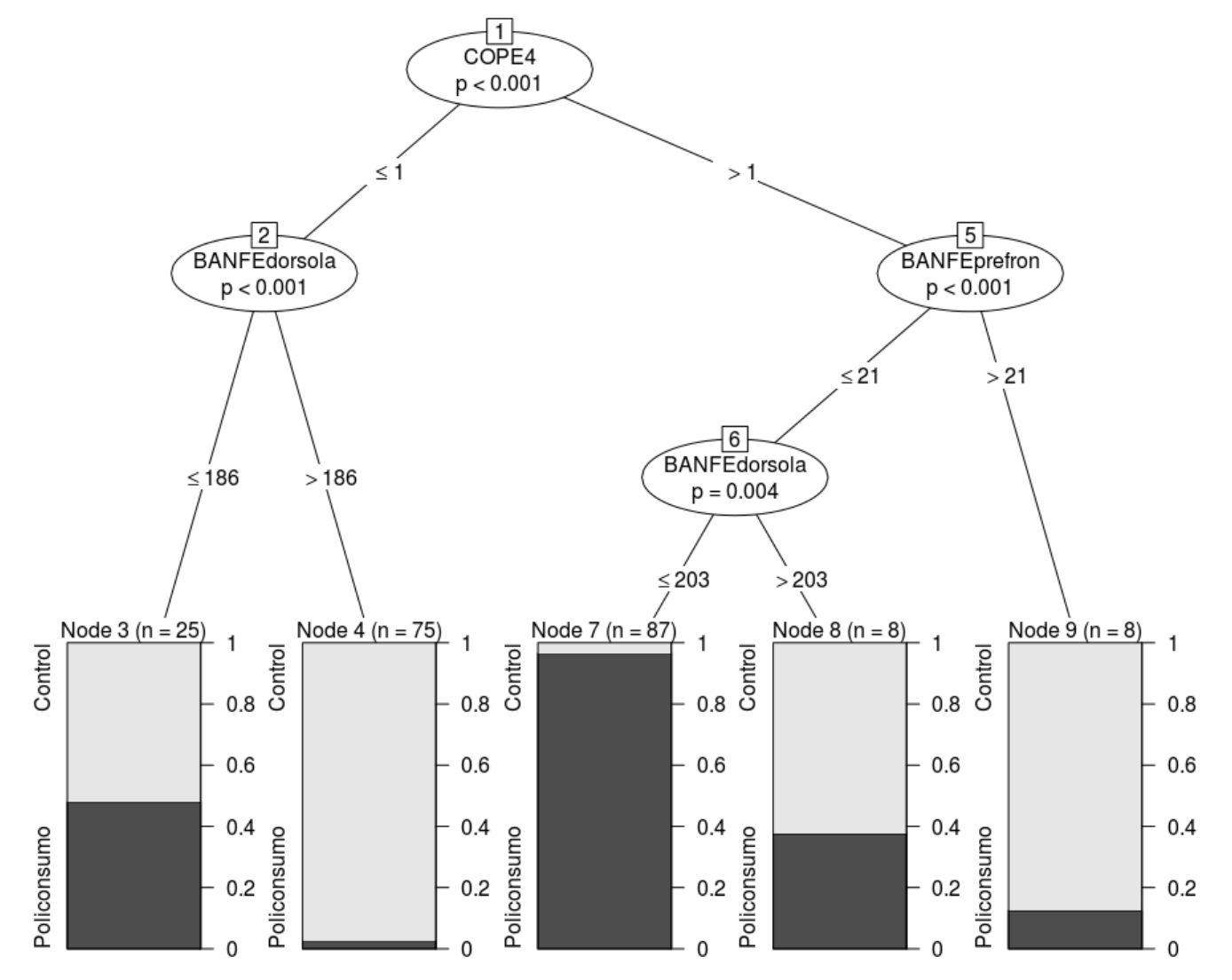

Figura 3. Random Forest para variables neuropsicológicas y clínicas

El resultado del análisis discriminante conjunto mostró una función significativa de separación máxima de los grupos $[\lambda=0,34, \mathrm{~F}(1,201)=379,9615, \mathrm{p}<0,05]$. La relación de la función aislada con los grupos fue de 0,80 (correlación canónica). Las variables con más peso a la hora de separar a los grupos fueron: Afrontamiento consumo de alcohol o drogas (COPE 4), BANFE total prefrontal, BANFE total dorsolateral, Impulsividad disfuncional e Índice de amplitud y diversidad psicopatológica (SCL90).

El análisis de especificidad y sensibilidad de las pruebas clínicas y neuropsicológicas a partir de las curvas ROC mostraron que la asignación de sujetos al grupo de policonsumidores (sensibilidad) es de un $88,84 \%$ en el uso de la variable Afrontamiento consumo de alcohol o drogas (COPE 4), seguida de las variables, BANFE total dorsolateral con un 88,77\%, BANFE total prefrontal con un 86,32, Impulsividad disfuncional 77,15 y, 
finalmente, el Índice global de amplitud y diversidad psicopatológica (SCL90). El estudio de estos contrastes mostró que tanto el afrontamiento centrado en el consumo de sustancias como la BANFE total dorsolateral fueron las variables que mejor pronosticaron la pertenencia a los grupos.

El análisis de regresión múltiple para estudiar el efecto del tiempo de consumo y la edad en las funciones ejecutivas y el ajuste clínico en el grupo de policonsumidores tuvo en cuenta las puntuaciones de la BANFE total funciones ejecutivas, siendo las variables predictoras la edad y el tiempo de consumo, así como el término de interacción Edad*Tiempo de Consumo. El análisis encontró el mejor efecto predictor al incluir ambas variables en el modelo, se observó un efecto significativo de dicha interacción Edad*Tiempo de Consumo $[\beta=0,42 ;$ ic $\left.(0,17-0,06) ; \mathrm{t}=3,46 ; \mathrm{p}<0,01 ; \mathrm{R}^{2}=12 \% ; \mathrm{F}(3,93)=5,44, \mathrm{p}<0,01\right]$. Dicho efecto de interacción se recoge en la figura 4.

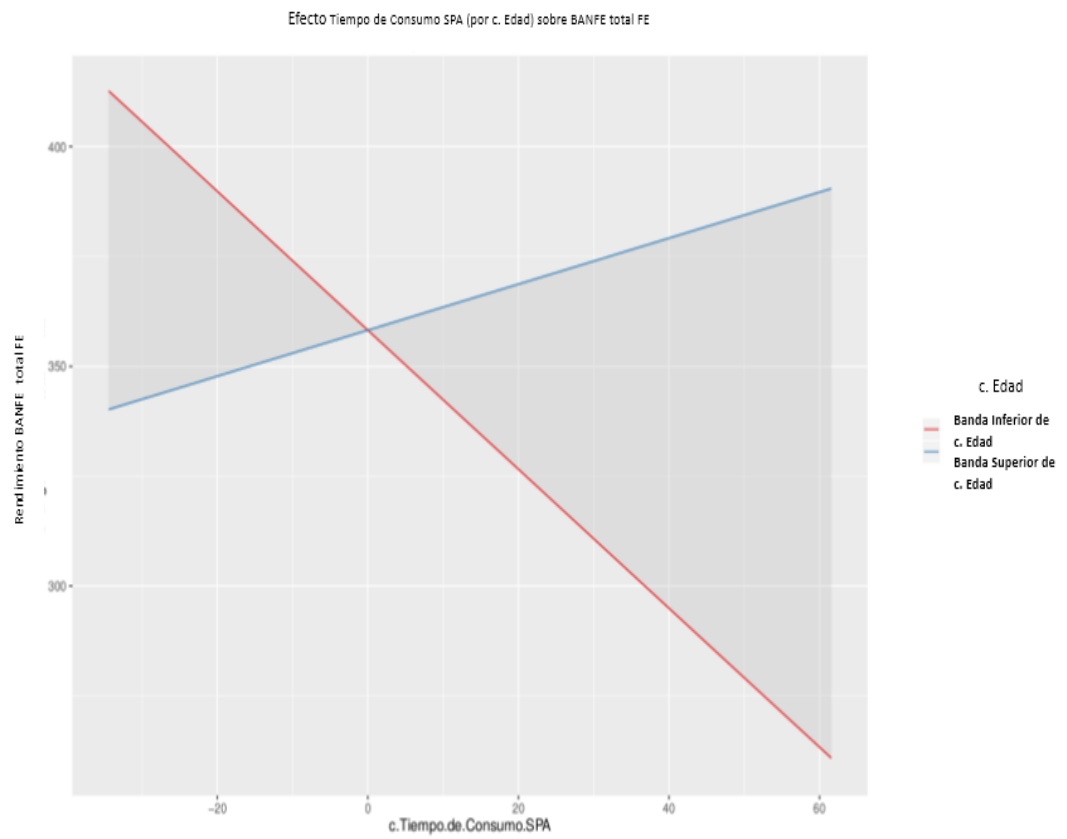

Figura 4. Interacción Tiempo de consumo y edad en la predicción del deterioro cognitivo

(BANFE total funciones ejecutivas).

El efecto observado en esta figura reflejaría que el grupo de policonsumidores más jóvenes (primer cuartil de la variable edad) presenta un decremento significativo en las puntuaciones de la BANFE total a medida que se incrementa el tiempo de consumo; patrón no observado en el grupo de policonsumidores de mayor edad, cuando tenemos en cuenta los mismos tiempos de consumo. Por tanto, podríamos concluir que la variable tiempo de consumo presenta un efecto diferencial determinado por la edad al estudiar el rendimiento en las funciones ejecutivas. Los análisis de regresión realizados para las variables clínicas relevantes no mostraron resultados significativos, indicando, por tanto, la ausencia de efecto de las variables edad y tiempo de consumo para dichas variables. 


\section{Discusión}

El presente estudio ha permitido establecer una comparación fiable entre el grupo objeto de estudio (policonsumidor) y el grupo control, dada la homogeneidad entre ambos en la características sociodemográficas. Este hecho constituye un avance con respecto a otras investigaciones, que en general adolecen de un menor rigor metodológico. Así mismo, el uso de la batería neuropsicológica de funciones ejecutivas y lóbulos frontales BANFE-2 permitió realizar una evaluación extensa y precisa del funcionamiento prefrontal en el grupo de adolescentes. De este modo, se logran evitar los sesgos observados en otros estudios en los que la evaluación de las funciones ejecutivas se lleva a cabo únicamente con pruebas y test aislados.

Los resultados obtenidos inicialmente, mostraron que el grupo de adolescentes policonsumidores obtuvo puntuaciones menores en las funciones ejecutivas vinculadas a las áreas prefrontal — anterior y dorsolateral一. Los procesos de abstracción y monitoreo, que se asocian a dominios de la corteza prefrontal anterior, reflejaron una menor ejecución entre los policonsumidores. La memoria de trabajo, la flexibilidad mental, procesamiento riesgo-benficio, la productividad verbal, la planificación y la inhibición, implicados en el funcionamiento de la corteza dorsolateral, también mostraron un menor rendimiento en este grupo. Estos resultados irían en la línea de trabajos de revisión realizados al respecto, como el de Fernández-Serrano, Pérez-García y Verdejo-García, (2011) en el que se identifica un conjunto común de alteraciones neuropsicológicas asociadas a la adicción, que con independencia de la sustancia implicada se centraría en un rendimiento ejecutivo deficitario.

Los resultados sobre el ajuste clínico mostraron diferencias significativas entre ambos grupos en los modos de afrontar el estrés, los niveles de autoestima, en impulsividad y en el índice de diversidad y amplitud psicopatológica. En relación a la autoestima, los resultados mostraron menores puntajes en el grupo de adolescentes policonsumidores, reflejando así, una mayor presencia de emociones negativas hacia sí mismos, en comparación con el grupo control. Estos resultados son coincidentes con las investigaciones que estudian dicha variable en adolescentes sanos y en consumidores de sustancias (Sánchez-Sosa, Villarreal-González, Ávila-Guerrero, Vera-Jiménez \& Musitu; Jiménez, 2011). Factores de riesgo como proceder de hogares uniparentales y familias reconstruidas, presencia de fracaso académico, altos niveles de ansiedad y consumo de sustancias por parte de miembros de la familia de policonsumidores se identificaron como variables con una negativa influencia sobre la autoestima del grupo de adolescentes consumidores.

Los hallazgos obtenidos en este estudio, resaltan la impulsividad disfuncional como una de las características clínicas representativas del grupo policonsumidor que refleja la tendencia a tomar decisiones irreflexivas, rápidas y sin precisión; orientando a este grupo de adolescentes al contacto temprano con las drogas, la perpetuación del consumo y la progresión hacia la adicción (Pedrero, López-Durán \& Olivar-Arroyo). En contraste, en el grupo control, se observó una mayor tendencia a tomar decisiones rápidas cuando la situación implica un beneficio personal, lo que supone un proceso de toma de decisiones con riesgo calculado. Según Dickman (1990), lo anterior caracteriza a la impulsividad funcional, que podría estar protegiendo al grupo control del contacto con las drogas.

En cuanto las estrategias de afrontamiento ante el estrés, los resultados del estudio, identifican el consumo de alcohol o drogas como una de los modos de afrontamiento más usado por la muestra de adolescentes policonsumidores y el único que se identificó en los análisis como de mayor peso a la hora de diferenciar los grupos. El uso de esta estrategia de afrontamiento para sentirse mejor o para pensar menos en los problemas y en el peor de los casos para intentar superarlos caracterizó al grupo policonsumidor en relación al grupo control; y revela la alta tendencia de este grupo de adolescentes, a reducir la tensión mediante el uso de sustancias incrementando las sensaciones placenteras, de bienestar, y socialización. Este estilo de afrontamiento pasivo y evitativo se torna en poco adaptativo e ineficaz, y suele predecir conductas autodestructivas y la vez se convierte en un factor de vulnerabilidad y riesgo para desencadenar el consumo de sustancias psicoactivas (Wong, Silva, Kecojevic, Schrager, Bloom, Iverson \& Lankenau, 2013).

La amplitud y diversidad psicopatológica es uno de los índices que mejor ha diferenciado al grupo policonsumidor del grupo control, identificándose síntomas propios de las nueve escalas sintomáticas. Los presentes resultados van en la línea de hallazgos similares sobre el tema. La mayoría de sustancias consumidas por el grupo policonsumidor se han asociado con una amplia presencia de síntomas psicopatológicos que, de acuerdo con la investigación, pueden generar cierta vulnerabilidad hacia el mantenimiento del consumo (Alves et al., 2014; Gómez-Rodríguez, Gutiérrez-Herrera \& Londoño, 2013). En la historia de consumo del grupo en mención 
se identificó la marihuana como una de las sustancias de mayor consumo a lo largo de la vida asociada en numerosos estudios a diversa sintomatología psicopatológica como depresión, trastorno bipolar, ansiedad y conducta suicida, entre otras (Borges, Bagge \& Orozco, 2016; Gómez-Rodríguez, Gutiérrez-Herrera \& Londoño, 2013); Lev-Ran, Le Foll, McKenzie, George \& Rehm, 2013).

Por lo que respecta al estudio de la influencia de la edad y el tiempo de consumo, los resultados mostraron un efecto predictivo de la interacción entre ambas variables sobre el rendimiento en las funciones ejecutivas y una ausencia de éste en las variables clínicas. Los adolescentes policonsumidores más jóvenes mostraron un decremento significativo del funcionamiento ejecutivo a medida que se incrementaba el tiempo de consumo; patrón que no fue observado en el grupo de mayor edad. Diversos estudios, mediante metodología longitudinal, han identificado el efecto de la edad en el declive cognoscitivo y los cambios en el sustrato neuroanatómico implicado en sujetos que iniciaron el consumo de sustancias (mayoritariamente cannabis y alcohol) en etapas iniciales de la adolescencia. Las diferencias estructurales más notables apuntan a mostrar el desequilibrio entre los estados de maduración de áreas corticales y subcorticales que se correlacionan con puntuaciones deficientes en pruebas neuropsicológicas (Bava, Frank, McQueeny, Schweinsburg, Schweinsburg, \& Tapert, 2009; Crane, Schuster, Fusar-Poli, \& Gonzalez, 2013; Gruber, Dahlgren, Sagar, Gönenç \& Lukas, 2014).

No obstante, en cierta medida los hallazgos del presente estudio, son compatibles con los observados en estos estudios si bien habría que tener en cuenta que no se estudió la relación edad y tiempo de consumo como en el presente caso y que las muestras utilizadas fueron heterogéneas, se emplearon pruebas aisladas y la mayoría se concentran en sustancias como alcohol y marihuana.

Los avances en la perspectiva biológica permiten hoy en día entender mejor la bioquímica de las sustancias psicoactivas y sus efectos; pero, sobre todo, sugieren que hay mecanismos subyacentes a las adicciones que, en el caso del cerebro adolescente, permiten entender su impacto en el desarrollo cerebral. Sin embargo, estos cambios provocados en la estructura y neuroquímica son condición necesaria, pero no suficiente, para entender el comportamiento compulsivo del adolescente. Factores como la disponibilidad de una familia estructurada y una red social adecuada, favorecerían el normal desarrollo, generando adolescentes más adaptativos y con un menor número de conductas de riesgo; indicando por tanto que los procesos de maduración neurológica no dependen exclusivamente de factores biológicos, sino de la constante interacción entre éstos y el ambiente que rodea al adolescente.

\section{Referencias}

Adan, A. (2012). Impulsividad funcional y disfuncional en jóvenes con consumo intensivo de alcohol (binge drinking). Adicciones, 24, 17-22. http://dx.doi.org/10.20882/adicciones.113

Acosta, J., Cervantes, M.L., Pineda, W.F., de la Torre, G., López, L., \& Cárdenas, B. (2011). Policonsumo desde una perspectiva neuropsicológica. Psicogente, 14, 178-189.

Alves, C.J., Magalhães, A., Melo, P., de Sousa, L., Tavares, M.A., Monteiro, P.R.R., \& Summavielle, T. (2014). Long-term effects of chronic cocaine exposure throughout adolescence on anxiety and stress responsivity in a Wistar rat model. Neuroscience, 277, 343-355. http://dx.doi.org/10.1016/j.neuroscience.2014.07.008

American Psychiatric Association, Diagnostic and Statistical Manual of Mental Disorders, (2000). DMS-IV. Manual diagnóstico y estadístico de los trastornos mentales $4^{\text {a }}$ edición. Washington DC: APA.

Bava, S., Frank, L.R., McQueeny, T., Schweinsburg, B.C., Schweinsburg, A.D., \& Tapert, S. F. (2009). Altered white matter microstructure in adolescent substance users. Psychiatry Research: Neuroimaging, 173, 228-237. http://dx.doi.org/10.1016/j.pscychresns.2009.04.005

Beckman, N., Anderson, K., Trim, R., Metrik, J., Diulio, A., Myers, M., \& Brown, S. (2011). Thinking and drinking: alcohol-related cognitions across stages of adolescent alcohol involvement. Psychology of Addictive Behaviors, 25, 415-425. http://dx.doi.10.1037/ a0023302

Blum, K., Femino, J., Teitelbaum, S., Giordano, J., Berman, M., \& Gold, M. (2013). Molecular Neurobiology Addiction Recovery. Nueva York: Springer. http://dx.doi.10.1007/978-1-4614-7230-8.

Bussey, M., Jørgensen, T., Pinnerup-Jensen, J., Bech-Jørgensen, K., Madsen, M., Nielsen, B., Nybo-Andersen, A.M., Søgaard-Nielsen A., \& Grønbaek, M. (2006). Influence of pretreatment coping strategies on the outcome of outpatient treatment of Danish alcohol abusers. European Addiction Research, 12, 83-90. http://dx.doi.10.1159/000090427 
Borges, G., Bagge, C.L., \& Orozco, R (2016). A literature review and meta-analyses of cannabis use and suicidality. Journal of Affective Disorders, 195, 63-74. http://dx.doi.org/10.1016/j.jad.2016.02.007

Breiman, L. (2001). Random forests. Machine learning, 45, 5-32. http://dx.doi:10.1023/A:1010933404324

Camacho, A. (2005). Factores Psicosociales relacionados con el consumo de sustancias psicoactivas en estudiantes de secundaria. Cuadernos Hispanoamericanos de Psicología, 5, 41-56.

Carver, C., Scheier, M., \& Weintraub, J. (1989). Assessing coping strategies: A theoretically based approach. Journal of Personality and Social Psychology, 56, 267-283. http://dx.doi.10.1037/0022-3514.56.2.267.

Corominas, M., Roncero, C., \& Casas, M. (2015). El Sistema Dopaminérgico en las Adicciones. Mente y Cerebro. Cuadernos, 10, 4-11.

Crane, N. A., Schuster, R. M., Fusar-Poli, P., \& Gonzalez, R. (2013). Effects of cannabis on neurocognitive functioning: recent advances, neurodevelopmental influences, and sex differences. Neuropsychology Review, 23, 117-137. http:/dx.doi.10.1007/s11065-012-9222-1

Crespo, M., \& Cruzado, J. (1997). La intervención del afrontamiento adaptación española del cuestionario COPE con una muestra de estudiantes universitarios. Análisis y Modificación de Conducta, 23, 797-830.

Dickman, S.J. (1990). Functional and dysfunctional impulsivity: Personality and cognitive correlates. Journal of Personality and Social Psychology, 58, 95-102. http://dx.doi.org/10.1037/0022-3514.58.1.95

Fernández-Serrano, M.J., Pérez-García, M., \& Verdejo-García, A. (2011). What are the specific vs. generalized effects of drugs of abuse on neuropsychological performance? Neuroscience \& Biobehavioral Reviews, 35, 377-406. http://dx.doi.org/10.1016/j.neubiorev.2010.04.008

Flores, E., \& Fernández, E. (2011). Neurociencia y Adicción. España: Sociedad Española de Toxicomanías.

Flores, J., Ostrosky, F., \& Lozano, A. (2014). BANFE-2, Batería Neuropsicológica de Funciones Ejecutivas y Lóbulos Frontales $2^{\mathrm{a}}$ Edición. México: Manual Moderno.

Gómez-Rodríguez, D.T., Gutiérrez-Herrera, M.G., \& Londoño, S.P. (2013). Depresión y consumo de sustancias psicoactivas en adolescentes en la ciudad de Bogotá. Psychologia: Avances de la disciplina, 7, 45-51.

Gullo, M.J., Loxton, N.J., \& Dawe, S. (2014). Impulsivity: Four ways five factors are not basic to addiction. Addictive Behaviors, 39, 1547-1556. http://dx.doi.org/10.1016/j.addbeh.2014.01.002

Gullo, M., \& Potenza, M. (2014). Impulsivity: Mechanisms, moderators and implications for addictive behaviors. Addictive Behavior, 39, 1543-1546. http://dx.doi.10.1016/j.addbheh.2014.06.005

Gruber, S.A., Dahlgren, M.K., Sagar, K.A., Gönenç, A., \& Lukas, S.E. (2014). Worth the wait: effects of age of onset of marijuana use on white matter and impulsivity. Psychopharmacology, 231, 1455-1465. http://dx.doi.10.1007/s00213-013-3326-z

Heitzeg, M.M., Cope, L.M., Martz, M.E., \& Hardee, J.E. (2015). Neuroimaging risk markers for substance abuse: recent findings on inhibitory control and reward system functioning. Current Addiction Report, 2, 91-103. http://dx.doi:10.1007/s40429-015-0048-9

Inozemtseva, O. (2013). La cognición en la conducta adictiva. En A. González y E. Matute (Eds.) Cerebro y Drogas. (pp. 31-45). México: Manual Moderno.

Jiménez, T.I., (2011). Autoestima de Riesgo y Protección: Una Mediación entre el Clima Familiar y el Consumo de Sustancias en Adolescentes. Psychosocial Intervention, 20, 53-61. http://dx.doi:10.5093/in2011v20n1a6

Konrad, K., Firk, C., \& Uhlhaas, P.J. (2013). Brain development during adolescence. Dtsch Arztebl Int, 110, 425-31. http://dx.doi:10.3238/ arztebl.2013.0425

Lev-Ran, S., Le Foll, B., McKenzie, K., George, T.P., \& Rehm, J. (2013). Bipolar disorder and co-occurring cannabis use disorders: Characteristics, co-morbidities and clinical correlates. Psychiatry Research, 209, 459-465. http://dx.doi.org/10.1016/j.psychres.2012.12.014

Llanero-Luque, M., Ruiz-Sánchez de León, J. M., \& Pedrero-Pérez, E. J. (2014). Intervención neuropsicofarmacológica en la adicción. En Ruiz-Sánchez de León, J. M. y Pedrero-Pérez, E. J. (eds.), Neuropsicología de la Adicción (pp. 169-180). Madrid: Panamericana.

Lydon, D., Wilson, S., Child, A., \& Geier, C. (2014). Adolescent brain maturation and smoking: What we know and where we' re headed. Neuroscience Behavioral Reviews, 45, 323-342. http://dx.doi.10.10167j.neubiorev.2014.07.003.

Mariño, N., Castro, J., \& Torrado, J. (2012). Funcionamiento ejecutivo en policonsumidores de sustancias psicoactivas. Revista de Psicología Universidad de Antioquia 4, 49-64.

Martinez, J., Amaya, W., Campillo, H., Rueda, G., Campo, A., \& Díaz, L. (2007). Consumo de sustancias psicoactivas en adolescentes, Bucaramanga, Colombia, 1996-2004. Revista de Salud Pública, 9, 215-229.

Ministerio de Justicia y del Derecho, Ministerio de Salud y protección social, Observatorio de Drogas de Colombia (2013). Estudio Nacional de Consumo de Sustancias Psicoactivas 2013 Informe Final.

Moral, M., \& Ovejero, A. (2011). Consumo abusivo de alcohol en adolescentes españoles: tendencias emergentes y percepciones de riesgo. Universitas Psychologica, 10, 71-87.

Muñoz, M.N., Gallego, C., Wartski, C.I., \& Álvarez, L.E. (2012). Familia y consumo de sustancias psicoactivas: una búsqueda de lo ausente. Index de Enfermería, 21, 136-140. http://dx.doi:org/10.4321/S1132-12962012000200006

Oliva, A. (2007). Desarrollo cerebral y asunción de riesgos durante la adolescencia. Apuntes de Psicología, 25, $239,254$. 
Páramo, M.A. (2011). Factores de riesgo y factores de protección en la adolescencia: Análisis de contenido a través de Grupos de Discusión. Terapia psicológica, 29, 85-95. https://dx.doi.org/10.4067/S0718-48082011000100009

Pedrero-Pérez, E.J. (2009). Evaluación de la impulsividad funcional y disfuncional en adictos a sustancias mediante el Inventario de Dickman. Psicothema, 21, 585-591.

Pedrero-Pérez, E.J., López-Durán, A., \& Olivar-Arroyo, A. (2011). Addiction: frontal personality change but not personality disorder comorbidity. Implications for treatment of addictive disorders. Personality traits theory, testing and influences, 1-36.

R Development Core Team (2008). R: A language and environment for statistica computing. R Foundation for Statistical Computing, Vienna, Austria. ISBN 3-900051-07-0, URL http://www.R-project.org.

Rosselló, J., \& Berríos-Hernández, M.N. (2004). Ideación suicida, depresión, actitudes disfuncionales, eventos de vida estresantes y autoestima en una muestra de adolescentes puertorriqueños/as. Interamerican Journal of Psychology, 38, 295-302.

Rosenberg, M. (1965). Society and the adolescent self-image. Princeton, NJ: Princeton University Press.

Rosenberg, P., \& Curtiss, L. (2014). Behavioral Adicction. Criteria, Evidence and Treatment. Estados Unidos: Elsevier.

Sajjadi, H., Harouni, G.G., \& Sani, M.S. (2015). Personal, Familial and Environmental Determinants of Drug Abuse: A Causal-Comparative Study. Global Journal of Health Science, 7, 367-374. https://doi.org/10.5539/gjhs.v7n4p367

Sánchez-Sosa, J.C., Villarreal-González, M.E., Ávila-Guerrero, M.E., Vera-Jiménez, A., \& Musitu, G. (2014). Contextos de socialización y consumo de drogas ilegales en adolescentes escolarizados. Psychosocial Intervention, 23, 69-78. https://doi.org/10.5093/in2014a7

Scott, R., Hides, L., Allen, S., \& Lubman, D. (2013). Coping style and ecstasy use motives as predictors of current mood symptoms in ecstasy users. Addictive Behavior, 38, 2465-2472. http://dx.doi.10.16/j.addbeh.2013.05.005

Stuss, D.T., \& Levine, B. (2002). Adult clinical neuropsychology: lessons from studies of the frontal lobes. Annual Review of Psychology, 53, 401-433. http://dx.doi.10.1146/annurev.psych.53.100901.135220

Thoma, P., Friedmann, C., \& Suchan, B. (2013). Empathy and social problem solving in alcohol dependence, mood disorders and selected personality disorders. Neuroscience and Biobehavioral Reviews. 37, 448-470. http://dx.doi.10.1016/j.neubiorev.2013.01.024

Todd, M., Tennen, H., Carney, M., Armeli, S., \& Affleck, G. (2004). Do we know how we cope? Relating daily coping reports to global and time-limited restrospective assessments. Journal of Personality and Social Psychology, 86, 310-319. http://dx. doi.10.10377/0022-3514.86.2.310

Vázquez-Morejón, R., Jiménez-García, M.R., \& Vázquez-Morejón, A.J. (2004). Escala de autoestima de Rosenberg: Fiabilidad y validez en población clínica española. Apuntes de Psicología, 22, 247-255.

Verdejo-García, A., Bechara, A., Recknor, E.C., \& Perez-Garcia, M. (2006). Executive dysfunction in substance dependent individuals during drug use and abstinence: an examination of the behavioral, cognitive and emotional correlates of addiction. Journal of the International Neuropsychological Society, 12, 405-415. http://dx.doi.org/10.1017/S1355617706060486

WHO ASSIST Working Group (2002). The alcohol, smoking and substance involvement screening test (ASSIST): development, reliability and feasibility. Addiction, 97, 1183-1194. http://dx.doi.10.1046/j.1360-0443.2002.00185.x

Wilson, A. G., Franck, C. T., Mueller, E. T., Landes, R. D., Kowal, B. P., Yi, R., \& Bickel, W. K. (2015). Predictors of delay discounting among smokers: Education level and a Utility Measure of Cigarette Reinforcement Efficacy are better predictors than demographics, smoking characteristics, executive functioning, impulsivity, or time perception. Addictive Behaviors, 45, 124-133. http://dx. doi:10.1016/j.addbeh.2015.01.027

Wong, C.F., Silva, K., Kecojevic, A., Schrager, S.M., Bloom, J.J., Iverson, E., \& Lankenau, S.E. (2013). Coping and emotion regulation profiles as predictors of nonmedical prescription drug and illicit drug use among high-risk young adults. Drug and Alcohol Dependence, 132, 165-171. http://dx.doi.org/10.1016/j.drugalcdep.2013.01.024 